\title{
European VLBI in limbo?
}

\section{The failure of an important and worthy application for research funds to the European Commission demonstrates that Europe needs a more imaginative way of backing research that matters.}

SOMETIMES, if rarely, there are research proposals that make perfect sense: the project is sound, indeed imaginative; the applicants have splendid track records, and are prepared to commit their own resources to the project; and there is every reason why the intended source of funds should welcome the proposal both as a vehicle for spending money wisely and because it furthers that organization's wider objectives. What executive officer of a grant-making organization could fail to jump at such a prize? Yet that is what the European Commission has just done.

The circumstances, which are not particularly complicated, concern the development of Very-Long-Baseline Interferometry (VLBI) in Europe. Everybody knows how valuable this technique has been shown to be in the past decade and a half. The principle is simply that of simultaneously pointing two or more radiotelescopes at the same distant radio source. The coordinated instruments then function as one instrument whose resolving power is proportional to the distances by which the instruments are separated (or, strictly, to the ratio of the baseline and the wavelength at which observations are made).

The principle is no different from that in which neighbouring radiotelescopes are deployed in concert to form a radiointerferometer, but there is a practical difference: because of the distances between the instruments, signals from different receivers cannot be compared in real time, but must instead be recorded on magnetic tape and correlated with each other later, using a device known as a correlator.

By now, the achievements of VLBI are widely appreciated. In the observation of distant radio galaxies, the technique promises the most direct observational evidence of what happens near the galaxysized nuclei of these often vastly extended objects, thus helping to decide whether the central galaxies are driven by black holes or by some other mechanism. Last year, an observation of the active nucleus of the radio galaxy $3 \mathrm{C} 84$ reported an accuracy of 100 microarcseconds (Bartel, N. et al. Nature 334, 131; 1988), no doubt still a record. But there are many more familiar astrophysical processes, such as the mapping of star-forming clouds of gas and dust within our galaxy and the study of the movement of matter near peculiar binary stars, which have been made intelligible by the use of VLBI.
All this was expected at the outset. The surprise has been the usefulness of VLBI in geodesy and geophysics. Coordinated radiotelescopes can so accurately measure the orientation of distant radio sources relative to the Earth's surface that VLBI measurements have become standard measures of the motion of the Earth's poles (including the Chandler Wobble) and of variations in the length of the day.

The point has also been reached at which VLBI is yielding, in what might be called real time, a log of the relative movement of the Earth's tectonic plates. The uncertainty in baseline measurements, inferred from the observation of known distant objects, is already no more than 3 $\mathrm{cm}$, comparable with the annual separation of the plates on either side of the MidAtlantic Ridge, and should be further reduced as more high-frequency instruments join the VLBI collaborations and as data analysis is improved. Already pairs of movable radiotelescopes have provided a plausible account of the internal distortion of the tectonic plate of the south-western United States.

But these developments are only a beginning. Coordinated observations by telescopes separated by the diameter of the Earth are commonplace, but now there are ambitions - even plans - to surmount the limitation of the Earth's finite size. The European and US space agencies have made a joint study of an orbiting radiotelescope, while there is a possibility that the Soviet Union may launch such a device in the early 1990s, as may Japan. VLBI, it seems, is booming.

That, sadly, is where recent European disappointments are ironical. From the outset, European radio-observatories have played an important part in wider international collaborations. Most of them now belong to a club, called the European VLBI consortium, which organises collaborations and worries about data acquisition and analysis.

There is the rub. For at least the past three years, it has been clear that existing facilities would soon be overwhelmed by data. Technically, there are two problems - that of recording data accurately in the expected volume (determined by the need to record with high time-resolution and over a range of frequencies) and that of comparing data from different stations so as to extract meaningful information.

This is what prompted the consortium last year to apply to the European Com- mission for funds with which to equip itself with suitable facilities. The hope had been that a correlator centre could be founded at the Westerbork observatory in the Netherlands, and that the Commission might also help equip individual stations with suitable recording equipment.

As might be expected, the Commission seems to have warmly applauded the proposal, as neat a marriage as there could be of scientific merit with the ideal of European collaboration. Much in the spirit in which censors about to ban books first read them through a second time, the Commission cannot be faulted for the attention which it has given the proposal. But the plan falls outside the terms of reference of the most relevant of the Community's research programmes, the Framework stimulation programme, which, administered by the CODEST committee, exists to bring European researchers together in research collaborations.

In grant-makers' terms, there are several pitfalls buried in the VLBI correlator project, not the least of which is that CODEST's own funds are repeatedly being overtaken by growing demands. But the VLBI correlator would also be a permanent institution, with annual running costs, of a kind that the Commission is not at present equipped (or even empowered) to shoulder. So everybody has been sympathetic, and CODEST has offered the consortium a grant of $300,000 \mathrm{ECU}$ (which it should accept) to improve on its proposal, or even to commission the design of a data-recorder.

This is a sad state of affairs for which nobody is to blame - as yet. While member states delight in playing cat-andmouse with the Commission over its science budget, it is natural that the Commission should shy from building institutions with implicit promises to support them. It is similarly natural that members of the consortium should obtain from national sources the equipment needed to remain in membership. And nobody can complain that the Commission should seek to make its funds go as far as possible by concentrating on "pump-priming seed-corn projects", to mix two grantmakers' metaphors.

But when, as on this occasion, it finds itself having to turn down a project of outstanding merit simply because its own rules are inappropriately designed, it should do the next most sensible thing and change the rules. 